Network Working Group

Request for Comments: 3152

R. Bush

BCP : 49

Updates: $2874,2772,2766,2553,1886$

RGnet

Category: Best Current Practice

\title{
Delegation of IP6.ARPA
}

Status of this Memo

This document specifies an Internet Best Current Practices for the Internet Community, and requests discussion and suggestions for improvements. Distribution of this memo is unlimited.

Copyright Notice

Copyright (C) The Internet Society (2001). All Rights Reserved.

Abstract

This document discusses the need for delegation of the IP6.ARPA DNS zone, and specifies a plan for the technical operation thereof.

1. WhY IP6.ARPA?

In the IPv6 address space, there is a need for 'reverse mapping' of addresses to DNS names analogous to that provided by the IN-ADDR.ARPA zone for IPV4.

The IAB recommended that the ARPA top level domain (the name is now considered an acronym for "Address and Routing Parameters Area") be used for technical infrastructure sub-domains when possible. It is already in use for IPv4 reverse mapping and has been established as the location for E.164 numbering on the Internet [RFC2916 RFC3026].

IETF consensus was reached that the IP6.ARPA domain be used for address to DNS name mapping for the IPv6 address space [RFC2874].

2. Obsoleted Usage

This document deprecates references to IP6.INT in [RFC1886] section 2.5, [RFC2553] section 6.2.3, [RFC2766] section 4.1, [RFC2772] section 7.1.C, and [RFC2874] section 2.5.

In this context, 'deprecate' means that the old usage is not appropriate for new implementations, and IP6.INT will likely be phased out in an orderly fashion. 


\section{IANA Considerations}

This memo requests that the IANA delegate the IP6.ARPA domain following instructions to be provided by the IAB. Names within this zone are to be further delegated to the regional IP registries in accordance with the delegation of IPv6 address space to those registries. The names allocated should be hierarchic in accordance with the address space assignment.

4. Security Considerations

While DNS spoofing of address to name mapping has been exploited in IPV4, delegation of the IP6.ARPA zone creates no new threats to the security of the internet.

5. References

[RFC1886] Thomson, S. and C. Huitema, "DNS Extensions to support IP version 6", RFC 1886, December 1995.

[RFC2553] Gilligan, R., Thomson, S., Bound, J. and W. Stevens, "Basic Socket Interface Extensions for IPv6", RFC 2553, March 1999.

[RFC2766] Tsirtsis, G. and P. Srisuresh, "Network Address Translation - Protocol Translation (NAT-PT)", RFC 2766, February 2000 .

[RFC2772] Rockell, R. and R. Fink, "6Bone Backbone Routing Guidelines", RFC 2772, February 2000.

[RFC2874] Crawford, M. and C. Huitema, "DNS Extensions to Support IPv6 Address Aggregation and Renumbering", RFC 2874, July 2001 .

[RFC2916] Faltstrom, P., "E.164 number and DNS", RFC 2916, September 2000 .

[RFC3026] Blane, R., "Liaison to IETF/ISOC on ENUM", RFC 3026, January 2001 . 
6. Author's Address

Randy Bush

5147 Crystal Springs

Bainbridge Island, WA US-98110

Phone: +1 2067800431

EMail: randyepsg.com 
Full Copyright statement

Copyright (C) The Internet Society (2001). All Rights Reserved.

This document and translations of it may be copied and furnished to others, and derivative works that comment on or otherwise explain it or assist in its implementation may be prepared, copied, published and distributed, in whole or in part, without restriction of any kind, provided that the above copyright notice and this paragraph are included on all such copies and derivative works. However, this document itself may not be modified in any way, such as by removing the copyright notice or references to the Internet society or other Internet organizations, except as needed for the purpose of developing Internet standards in which case the procedures for copyrights defined in the Internet Standards process must be followed, or as required to translate it into languages other than English.

The limited permissions granted above are perpetual and will not be revoked by the Internet society or its successors or assigns.

This document and the information contained herein is provided on an "AS IS" basis and THE INTERNET SOCIETY AND THE INTERNET ENGINEERING TASK FORCE DISCLAIMS ALL WARRANTIES, EXPRESS OR IMPLIED, INCLUDING BUT NOT LIMITED TO ANY WARRANTY THAT THE USE OF THE INFORMATION HEREIN WILL NOT INFRINGE ANY RIGHTS OR ANY IMPLIED WARRANTIES OF MERCHANTABILITY OR FITNESS FOR A PARTICULAR PURPOSE.

Acknowledgement

Funding for the RFC Editor function is currently provided by the Internet society. 